\title{
Individualized prediction of psychosis in subjects with an at-risk mental state
}

Eleni Zarogianni ${ }^{1}$, Amos J. Storkey ${ }^{2}$, Stefan Borgwardt ${ }^{3}$, Renata Smieskova ${ }^{3}$, Erich Studerus ${ }^{4}$, Anita Riecher-Rössler ${ }^{4}$, Stephen M. Lawrie ${ }^{1}$

${ }^{1}$ Division of Psychiatry, School of Clinical Sciences, University of Edinburgh, the Royal Edinburgh Hospital, Morningside Park, UK

${ }^{2}$ Institute for Adaptive and Neural Computation, University of Edinburgh, UK

${ }^{3}$ Department of Psychiatry (UPK), University of Basel, Switzerland

${ }^{4}$ Center for Gender Research and Early Detection, University of Basel Psychiatric

Hospital, Switzerland

Correspondence to: Eleni Zarogianni, Kennedy Tower, Division of Psychiatry, School of Clinical Sciences, University of Edinburgh, the Royal Edinburgh Hospital, Morningside Park, EH10 5HF, UK. E-mail: ezarogia@ exseed.ed.ac.uk. Tel.: +44(0) 1315376182.

Highlights: Our neuroanatomical-based classifier is able to predict transition to psychosis in individuals with an at-risk mental state. Replication of previous classification findings where a cohort of familial high-risk individuals was used.

Keywords: early diagnosis, psychosis onset, magnetic resonance imaging, support vector machine, at-risk mental state.

\section{Abstract}

Early intervention strategies in psychosis would significantly benefit from the identification of reliable prognostic biomarkers. Pattern classification methods have shown the feasibility of an early diagnosis of psychosis onset both in clinical and familial high-risk populations. Here we were interested in replicating our previous classification findings using an independent cohort at clinical high risk for psychosis, drawn from the prospective FePsy (Fruherkennung von Psychosen) study. The same neuroanatomicalbased pattern classification pipeline, consisting of a linear Support Vector Machine (SVM) and a Recursive Feature Selection (RFE) achieved 74\% accuracy in predicting later onset of psychosis. The discriminative neuroanatomical pattern underlying this 
finding consisted of many brain areas across all four lobes and the cerebellum. These results provide proof-of-concept that the early diagnosis of psychosis is feasible using neuroanatomical-based pattern recognition.

\section{Introduction}

Over the past 10 years, the target of early intervention strategies has shifted from the early diagnosis of first episode of psychosis (Perkins et al. 2005, Marshall et al. 2005) towards the early identification and treatment of individuals exhibiting early signs of psychosis. Early detection and intervention centres, such as the Personal Assessment and Crisis Evaluation (PACE) in Australia (Yung et al. 1996), the Prevention through Risk Identification, Management and Education (PRIME) in the United States (McGlashan et al. 2003), and the Outreach and Support in South London (OASIS) clinic (Fusar-Poli et al. 2013) have been set up worldwide, aiming to provide case management and provisional treatment for individuals presenting with sub-threshold psychotic symptoms and/or a decline in functioning. Individuals with this clinical presentation are considered at increased risk for developing psychosis and are named as ultra high-risk (UHR) individuals or at an at-risk mental state (ARMS).

The rationale for an ARMS stems from observations that psychosis and schizophrenia begin many years before the emergence of frank, psychotic symptoms, with nonspecific changes, perceptual alterations and often attenuated or transient psychotic disturbances (Riecher-Rössler et al. 2006). Currently, the identification of an ARMS relies on operationalized criteria that are based on a combination of trait and state risk factors (Yung et al. 2008, Yung et al. 1998). The predictive validity of these operationalized tools is however low, as transition rates show significant variation across clinical HR sites and recent studies reported a decline in the transition risk (Yung et al. 2007).

Reliable prognostic biomarkers could complement current assessment tools and also aid in improving the prediction of conversion and reducing the number of false positives. A series of putative biomarkers have been recently identified, suggesting that the ARMS is characterized by abnormalities in the neurocognitive domain (Lencz et al. 2006, Fusar- 
Poli et al. 2012a, Koutsouleris et al. 2012a) and alterations at the neuroanatomical (FusarPoli et al. 2011, Smieskova et al. 2010, Mechelli et al. 2011, Dazzan et al. 2012) and neurofunctional level (Fusar-Poli et al. 2007). Conversion to psychosis in ARMS subjects has been associated with reduced grey matter volume in the prefrontal and temporal cortices and other subcortical brain structures (Smieskova et al. 2010, Borgwardt et al. 2007a, Borgwardt et al. 2007b, Borgwardt et al. 2008, Pantelis et al. 2003, Koutsouleris et al. 2009, Mechelli et al. 2011, Dazzan et al. 2012, Fusar-Poli et al. 2011).

Recently, multivariate pattern recognition approaches, including SVM, have provided important leads towards the translation of neuroimaging findings into clinical practice, by taking into account inter-regional correlations between brain regions and working at the single-subject level (Orru et al. 2012, Zarogianni et al. 2013). These methods may thus provide the means for an individualized risk assessment and prediction of psychosis conversion and possibly deliver increased sensitivity and specificity, both of which are essential for informing individualized prevention care (Phillips et al. 2006).

Previous machine learning studies have shown that a neuroanatomical-based prediction of psychosis is possible at the single-subject level (Koutsouleris et al. 2009, Borgwardt et al. 2013), providing diagnostic accuracy up to 85\% (Koutsouleris et al. 2012b, Koutsouleris et al. 2015). Based on our previous study (Zarogianni et al. 2016), we have shown that a linear MRI-based SVM classifier can predict with significant accuracy the later transition to schizophrenia in a cohort of familial high-risk individuals. Here, we aimed to examine the generalizability of our classification method in predicting transition to psychosis using baseline structural MRI data from an independent cohort of subjects with an ARMS.

\section{Materials and Methods}

\subsection{Subjects}

Subjects included in this analysis were part of the prospective, early psychosis, FePsy 
(Fruherkennung von Psychosen) study. Recruitment and screening strategies have been described in detail previously (Riecher-Rössler et al. 2006, Riecher-Rössler et al. 2007). Briefly, subjects were recruited through a specialized clinic for the early detection of psychosis at the Psychiatric Outpatient Department, University Hospital in Basel, Switzerland. All aspects of the study were approved by the Ethics Committee of Basel and written informed consent was obtained from each participant before study inclusion.

Subjects were initially screened using the Basel Screening Instrument for Psychosis (BSIP, Riecher-Rössler et al. 2008), which is a 46-item checklist based on variables reported as risk factors or predictors of psychosis (Riecher-Rössler et al. 2006, RiecherRössler et al. 2007). For psychosis items, the Brief Psychiatric Rating Scale (BPRS; expanded version (Ventura et al. 1993)) for assessing (pre)-psychotic phenomena were incorporated. The BSIP allows the rating of individuals regarding the inclusion/exclusion criteria, corresponding to the PACE criteria (Yung et al. 1998), and has been shown to have a good interrater reliability $(\kappa=.67)$ for the assessment of the main outcome category "at risk for psychosis" and a high predictability (Riecher-Rössler et al. 2008).

Subjects were identified as at an ARMS if they manifested one (or more) of the following criteria, corresponding to the widely used PACE criteria (Yung et al. 1998): i) presence of attenuated psychotic-like symptoms (APS), ii) brief limited intermittent psychotic symptoms (BLIPS) or iii) a genetic risk of psycosis plus at least 2 further risk factors according to the BSIP checklist. Table 1 provides a detailed description of the inclusion requirements of the ARMS group.

Additionally, negative symptomatology was assessed using the Scale for the Assessment of Negative Symptoms (SANS; Andreasen 1989), in combination with the BSIP. Briefly, the SANS assessment is a well-recognised rating scale that consists of 19 items assessing negative symptoms of psychosis, which are further grouped into five domains (affective flattening, alogia, avolition-apathy, anhedonia-asociality, and inattention). Exclusion criteria included age below 18 years, insufficient knowledge of German, IQ <70, previous episodes of schizophrenic psychosis (treated with major tranquillizers for more than 3 weeks), a clearly diagnosed brain disease or substance dependency (except for cannabis dependency), or psychotic symptoms within a clearly diagnosed depression or borderline 
personality disorder.

Study inclusion started in March 2000 and continued until February 2004. Individuals were followed up at monthly intervals during the first year, at 3-month intervals during the second and third year and annually thereafter until transition to psychosis was established or until the end of the follow-up period (2007). In general, all ARMS subjects were followed up for over 4 years during which they were also offered supportive counselling and clinical management.

In total, 37 ARMS individuals were recruited. Thirty of the 37 ARMS individuals never received antipsychotic medication while 7 participants were administered low doses of antipsychotic medication for behavioural control (2 participants on olanzapine, 2 Chlorprothixene and 3 risperidone) prior to study inclusion, all for less than 3 weeks.

Matched groups of healthy controls and first-episode patients were recruited as well. In short, 22 healthy controls (HC) with no history of any psychiatric disorder and 25 firstepisode (FE) individuals, who met operationalized criteria for first-episode psychosis as described in Yung et al. 1998, were recruited.

Transition to psychosis was operationally defined by meeting PACE criteria (Yung et al. 1998 - Table 1) and further determined by a diagnostic interview using the ICD-10 criteria at the time of transition. Follow-up information for 2 ARMS subjects was not available. In this regard, 16 of the 35 ARMS individuals with retained follow-up information made a transition to psychosis (ARMS-T) and 19 did not convert (ARMSNT).

Here, we were interested in contrasting the ARMS group that converted to psychosis (ARMS-T) against the ARMS group that did not (ARMS-NT; Table 2). 


\subsection{Image Acquisition and Preprocessing}

Subjects were scanned using a Siemens (Erlangen, Germany) Magnetom Vision 1.5 T scanner at the University Hospital Basel. Head movement was minimized by foam padding and velcro straps across the forehead and chin. A three-dimensional volumetric spoiled gradient recalled echo sequence generated 176 contiguous, $1 \mathrm{~mm}$ thick sagittal slices. Imaging parameters were: time-to-echo, $4 \mathrm{msec}$; time-to-repetition, $9.7 \mathrm{msec}$; flip angle, 12; matrix size, 200 x 256; field of view, 25.6 x $25.6 \mathrm{~cm}$ matrix; voxel dimensions, $1.28 \times 1 \times 1 \mathrm{~mm}$.

Standard VBM procedures (Ashburner and Friston, 2000) were followed using SPM5 (http://www.fil.ion.ucl.ac.uk/spm/). Study-specific templates and customized prior probability maps were constructed using data from all study groupds in order to represent the entire study population and therefore minimise bias for spatial normalization. The scans were normalised to the generic SPM T1 template using 12-point linear affine transformation to minimise the residual sum of squares differences between the images and the template. A study-specific T1 template was created from the mean image calculated from all the normalised T1 images and smoothed at 8-mm full-width at half maximum (FWHM). To generate study-specific brain tissue a priori maps, the normalized images were segmented and then mean images for the normalized GM, WM and CSF segments were produced and finally smoothed at $8-\mathrm{mm}$ full-width at half maximum (FWHM).

Then, the baseline $\mathrm{T} 1$ scans entered the same pre-processing pipeline, described previously (Zarogianni et al. 2016). Briefly, T1 brain scans were segmented in native space into gray matter (GM), white matter (WM) and cerebrospinal fluid (CSF) and study-specific images for GM, WM and CSF, after which the SPM brain extraction function returned a tissue mask for each scan. These masks were, then, applied to the original $\mathrm{T} 1$ images to remove non-brain tissue. $\mathrm{T} 1$ brain images were, then, spatially normalized to the study-specific T1 template, using a 12-parameter linear affine transformation. Bilinear interpolation was used to resample the normalized images and write MNI-normalized images into the stereotactic space at a $1 \times 1 \times 1 \mathrm{~mm}$ voxel 
resolution. Normalized images were again segmented using study-specific a priori templates and spatially normalized segments for GM, WM and CFS were returned. Finally, the spatially normalized, segmented imageswere smoothedwith an 8mmfullwidth at maximum (FWHM) isotropic Gaussian kernel.

\subsection{Multivariate Pattern Classification Analysis}

\subsubsection{Support Vector Machine}

The SVM is a multivariate pattern recognition technique that has been widely used in neuroimaging-based studies because it can provide optimal decision rules for classification. Here, a linear SVM classifier was used for the classification task because it allows the straightforward extraction of the corresponding discrimination map. A detailed description of the SVM was given in our previous work (Zarogianni et al. 2016).

\subsubsection{Feature Extraction}

Prior to SVM, all smoothed and normalized GM maps were mapped to the Automated Anatomical Labeling (AAL) brain atlas (Tzourio-Mazoyer et al., 2002) and GM density volumes corresponding to the 116 brain regions of the template were returned. Features in the training set were also scaled to the [0 1$]$ template before applying the same normalization template to the testing set.

\subsubsection{Recursive Feature Elimination}

To identify the most significant features in the classification task and simultaneously increase classification performance, the recursive feature elimination (RFE) method (Guyon et al., 2002) was embedded in a nested leave-one-out cross-validation (LOO$\mathrm{CV}$ ), as described in Figure 1 and previously (Zarogianni et al. 2016).

\subsubsection{Permutation testing}

Permutation testing was performed in order to derive a $p$ value for the accuracy of our classifier. We permuted the class labels 1000 times (randomly assigning patient and control labels to the training subjects) and repeated the entire nested LOO-CV procedure. 
We then calculated the number of times in which the specificity (percentage of true negative) and sensitivity (percentage of true positive) for the permuted labels were higher than those obtained for the real labels. Dividing this number by 1000 we derived a $p$ value for the classification accuracies.

\subsubsection{Discrimination map}

A discrimination map was again generated based on the weight coefficients of the features that were selected by the RFE method (Figure 2). The discrimination map consists of brain regions that according to the RFE methodology are the most distinctive in the classification task and provides a spatial representation of the decision function in that every feature contributes with a certain weight to this function (or hyperplane). The SVM weight vector is a linear combination or weighted average of the support vectors and defines the decision boundary. The weight vector is therefore a spatial representation of the decision boundary. Every feature contributes with a certain weight to the decision boundary or classification function. Given a positive and a negative class $(+1=$ ARMS-T; -1=ARMS-NT group), a positive weight means the weighted average in that region was higher for the ARMS-T group, and a negative weight means the weighted average was higher for ARMS-NT group. Since the SVM classifier is multivariate by nature, it should be noted that all brain regions constituting the decision function contribute to the classification.

\section{Results}

\subsection{Socio-demographic and clinical findings}

The rate of conversion to psychosis was $45.7 \%$ in this ARMS sample of 35 subjects. The mean interval between the baseline scan and disease conversion was 306 days (median: 263, range: 25-1137 days). There were no significant differences between converters and non-converters to psychosis with regards to age, gender, educational level, verbal IQ, cannabis use at study entry, baseline global BPRS and SANS scores (Table 2). However, 
there was a significant difference in the two groups in the use of antipsychotics, with 6 ARMS-T subjects and 1 AMRS-NT having taken neuroleptics some time before study inclusion (Table 2).

\subsection{SVM classification analysis}

The application of our LOO-CV SVM-RFE methodology to baseline structural MRI data of the ARMS groups achieved $74 \%$ accuracy in predicting conversion to psychosis (Table 3). Six out of 16 subjects in the ARMS-T group were wrongly classified as ARMS-NT, while only 3 out of 19 subjects in the ARMS-NT group were incorrectly labeled as ARMS-T (sensitivity/specificity: 63\%/84\%; PPV/NPV: 77\%/73\%; permutation test $\mathrm{p}=0.002$ ).

The likelihood ratio of a positive test result was $\mathrm{LR}+=3.95$ (Table 6.3), meaning that a positive prognostic test in a given ARMS subject would increase the probability of a subsequent transition to psychosis from $45.7 \%$ to $77 \%$ (posttest probability=posttest odds/posttest odds+1, posttest odds=pretest odds*LR+).

The misclassified ARMS-NT subjects did not significantly differ from the correctly classified ARMS-NT in any of the socio-demographic or clinical variables (Table 4). On the contrary, the misclassified ARMS-T subjects were significantly different from the correctly classified ARMS-T individuals in terms of gender distribution and use of antipsychotic medication before study entry (Table 4). This may partly explain the lower sensitivity of the SVM-RFE method, since the ARMS-T group consisted of a more inhomogenous group of individuals with regards to anti-psychotic medication, which in turn might have hindered the identification of a common neuroanatomical signature across subjects in this group. The effect of antipsychotic medication in brain structure is widely acknowledged by the scientific community (Smieskova et al. 2009, Navari and Dazzan 2009), and might have played a major role in the classification of the ARMS subjects here, despite the fact the exposure was before study entry and relatively brief. 
The spatially distributed network that discriminated between the two groups was quite extensive and consisted of GM abnormalities in a spatially distributed network covering all four lobes and the cerebellum. Table 5 presents the most discriminating regions in the classification task, namely the brain regions with the highest (absolute) weight value that contributed relatively higher to the decision function. Specifically, the regions that contributed more in the classification of the ARMS-T subjects included the cerebellum, parts of the superior temporal pole bilaterally, the right anterior cingulate cortex, the right superior medial frontal and left orbitofrontal cortex and the insula bilaterally, whereas regions with a higher weighted average for the ARMS-NT group consisted of the right inferior parietal lobe, right medial temporal lobe, the right orbitofrontal cortex and the left pallidum.

A discrimination map showing the spatial pattern by which the groups differ is also illustrated in Figure 2. We emphasize that this spatially distributed pattern should not be interpreted as a statistical map, but rather as a spatial representation of the decision boundary.

\section{Discussion}

The present findings replicate our previous ones in that MRI-based classification methods were able to predict transition to psychosis in subjects at high clinical risk for developing the disorder using neuroanatomical data at study inclusion. The SVM-RFE classifier achieved $74 \%$ accuracy in classifying ARMS-T against ARMS-NT subjects.

The neuroanatomical decision function that discriminated the two groups was associated with GM abnormalities relying on a distributed network of regions covering most cortical and sub-cortical brain structures and the cerebellum. Our present findings agree with findings from a recent voxel-based meta-analysis that reported GM volume reductions in subjects that convert to psychosis in the insular and superior temporal lobe cortices (Fusar-Poli et al. 2011) and also with previous VBM findings on the same dataset 
(Borgardt et al. 2007a).

Despite being significant, our classification accuracy here is lower than the accuracy observed in our familial high-risk group (Zarogianni et al. 2016) where the same classification pipeline was used. Additionally, previous studies using the same ARMS cohort achieved higher classification performances than reported accuracies here (Koutsouleris et al. 2012b, Koutsouleris et al. 2015).

In the studies conducted by Koutsouleris and colleagues, (2012a and 2015), their MRIbased classifier achieved $84.2 \%$ and $75 \%$ accuracy respectively, in correctly classifying ARMS-T against ARMS-NT individuals drawn from the FePsy study. Differences in the observed classification accuracies may be partly explained by differences in the preprocessing of the MRI scans (where RAVENS maps and the VBM 8 toolbox were used) and partly by the chosen implementation of the SVM classifier, which relied upon the construction of SVM ensembles that incorporated feature selection, model training and predictive learning wrapped together in a repeated nested cross-validation framework. Ensemble learning approaches are usually selected on the basis that they can achieve higher predictive performance than single classifiers, by combining multiple weak learning models that decide upon the classification of a new instance through majority voting (Polikar 2006).

Compared to the diagnostic performance of our classifier in the familial high-risk cohort of the Edinburgh High Risk Study, the classification performance in the ARMS groups of the FePsy study was notably lower, contrary to what would be expected since the ARMS groups represent help-seeking individuals, most of whom already manifest putative transient and/or sub-threshold psychotic symptoms. Interestingly, 5 out of the 6 ARMS-T subjects that were misclassified received anti-psychotic medication some time before study inclusion (Table 4) while the other misclassified ARMS-T subject was prescribed tranquilizers (Lorazepam). Many studies have reported the effect of antipsychotic medication on grey matter volume in the direction of significant regional reductions (Navari and Dazzan 2009, Smieskova et al. 2009), thus possibly suggesting a neuroanatomical heterogeneity expressed with divergent pathophysiological trajectories 
between subjects receiving and subjects not receiving any anti-psychotic treatment.

Despite the lower diagnostic performance, our MRI-based classifier managed to increase the diagnostic certainty from $45.7 \%$ to $77 \%$ in case of a positive test result, suggesting that an MRI-based pattern classification system could, with refinement, become a useful part of a multi-step diagnostic procedure that would reliably quantify the risk for conversion to psychosis and inform appropriate care and treatment strategies.

Certain limitations of this study have to be considered. Again the sample size in this investigation is small. The rate of transition to psychosis amounted to nearly $46 \%$, which is generally in keeping with other clinically at-risk cohorts (Koutsouleris et al. 2009, Yung et al. 2003, Klosterkotter et al. 2001). However, it is not clear how the classifier would perform if presented with an ARMS cohort with significantly lower conversion rates. Finally, the administration of antipsychotic and antidepressant medication might have confounded our results, despite the fact that any drug treatment was administered before study inclusion. 


\section{References}

Ashburner, J., Friston, K.J., 2000. Voxel-based morphometry-the methods. NeuroImage 11 (6 Pt 1), 805-821.

Andreasen NC, 1989. The Scale for the Assessment of Negative Symptoms (SANS): Conceptual and theoretical foundations. Br J Psychiatry Suppl, 7:49 -58.

Borgwardt SJ, McGuire PK, Aston J, et al., 2007a. Structural brain abnormalities in individuals with an at-risk mental state who later develop psychosis. Br J Psychiatry Suppl, 51:s69-75.

Borgwardt SJ, Riecher-Rössler A, Dazzan P, et al., 2007b. Regional gray matter volume abnormalities in the at risk mental state. Biol. Psychiatry, 61(10):1148-1156.

Borgwardt SJ, McGuire PK, Aston J, et al., 2008. Reductions in frontal, temporal and parietal volume associated with the onset of psychosis. Schizophrenia Research, 106(23):108-114.

Borgwardt S, Koutsouleris N, Aston J, et al., 2013. Distinguishing prodromal from firstepisode psychosis using neuroanatomical single-subject pattern recognition. Schizophr Bull, 39(5):1105-1114..

Dazzan P, Soulsby B, Mechelli A, et al., 2012. Volumetric abnormalities predating the onset of schizophrenia and affective psychoses: an MRI study in subjects at ultra high risk of psychosis. Schizophr Bull, 38(5):1083-1091.

Fusar-Poli P, Perez J, Broome M, et al., 2007. Neurofunctional correlates of vulnerability to psychosis: a systematic review and meta-analysis. Neurosci Biobehav Rev, 31(4):465484.

Fusar-Poli P, Borgwardt S, Crescini A, et al., 2011. Neuroanatomy of vulnerability to psychosis: a voxel-based meta-analysis. Neurosci Biobehav Rev, 35(5):1175-1185.

Fusar-Poli P, Deste G, Smieskova R, et al., 2012a. Cognitive functioning in prodromal psychosis: a meta-analysis. Arch Gen Psychiatry, 69(6):562-571.

Fusar-Poli P, Bonoldi I, Yung AR, et al., 2012b. Predicting psychosis: meta-analysis of transition outcomes in individuals at high clinical risk. Arch. Gen. Psychiatry, 69(3):220229.

Fusar-Poli P, Byrne M, Badger S, et al., 2013. Outreach and support in south London (OASIS), 2001-2011: ten years of early diagnosis and treatment for young individuals at high clinical risk for psychosis. Eur. Psychiatry, 28(5):315-326.

Guyon, I., Weston, J., Barnhill, S, et al., 2002. Gene selection for cancer classification using support vector machines. Mach. Learn. 46 (1-3), 389-422.

Klosterkötter J, Hellmich M, Steinmeyer EM, et al., 2001. Diagnosing schizophrenia in the initial prodromal phase. Arch. Gen. Psychiatry, 58(2):158-164.

Koutsouleris N, Meisenzahl EM, Davatzikos C, et al., 2009. Use of neuroanatomical pattern classification to identify subjects in at-risk mental states of psychosis and predict disease transition. Arch. Gen. Psychiatry, 66(7):700-712. 
Koutsouleris N, Davatzikos C, Bottlender R, et al., 2012a. Early recognition and disease prediction in the at-risk mental states for psychosis using neurocognitive pattern classification. Schizophr Bull, 38(6):1200-1215.

Koutsouleris N, Borgwardt S, Meisenzahl EM, et al., 2012b. Disease prediction in the atrisk mental state for psychosis using neuroanatomical biomarkers: results from the FePsy study. Schizophr Bull, 38(6):1234-1246.

Koutsouleris N, Riecher-Rössler A, Meisenzahl EM, et al., 2015. Detecting the psychosis prodrome across high-risk populations using neuroanatomical biomarkers. Schizophr Bull, 41(2):471-482.

Lencz T, Smith CW, McLaughlin D, et al., 2006. Generalized and specific neurocognitive deficits in prodromal schizophrenia. Biol. Psychiatry, 59(9):863-871.

Lukoff D, Nuechterlein KH, Ventura J. 1986. Manual for the expanded brief psychiatric rating scale. Schizophr Bull, 12:594-602.

Marshall M, Lewis S, Lockwood A, et al., 2005. Association between duration of untreated psychosis and outcome in cohorts of first-episode patients: a systematic review. Arch. Gen. Psychiatry, 62(9):975-983.

McGlashan TH, Zipursky RB, Perkins D, et al., 2003. The PRIME North America randomized double-blind clinical trial of olanzapine versus placebo in patients at risk of being prodromally symptomatic for psychosis. I. Study rationale and design. Schizophr. Res, 61(1):7-18.

Mechelli A, Riecher-Rössler A, Meisenzahl EM, et al., 2011. Neuroanatomical abnormalities that predate the onset of psychosis: a multicenter study. Arch. Gen. Psychiatry, 68(5):489-495.

Orrù G, Pettersson-Yeo W, Marquand AF, et al., 2012. Using Support Vector Machine to identify imaging biomarkers of neurological and psychiatric disease: a critical review. Neurosci Biobehav Rev, 36(4):1140-1152.

Pantelis C, Velakoulis D, McGorry PD, et al., 2003. Neuroanatomical abnormalities before and after onset of psychosis: a cross-sectional and longitudinal MRI comparison. Lancet, 361(9354):281-288.

Perkins DO, Gu H, Boteva K, et al., 2005. Relationship between duration of untreated psychosis and outcome in first-episode schizophrenia: a critical review and metaanalysis. Am J Psychiatry, 162(10):1785-1804.

Phillips KA, Van Bebber S, Issa AM. 2006. Diagnostics and biomarker development: priming the pipeline. Nat Rev Drug Discov, 5(6):463-469.

Polikar, R. 2006. Ensemble based systems in decision making. IEEE Circuits and Systems Magazine, 6(3), 21-45. doi:10.1109/MCAS.2006.1688199

Riecher-Rössler A, Gschwandtner U, Borgwardt S, et al., 2006. Early detection and treatment of schizophrenia: how early? Acta Psychiatr Scand Suppl, 429:73-80. 
Riecher-Rössler A, Gschwandtner U, Aston J, et al., 2007)]. The Basel early-detectionof-psychosis (FEPSY)-study--design and preliminary results. Acta Psychiatr Scand, 115(2):114-125.

Riecher-Rössler A, Aston J, Ventura J, et al., 2008. [The Basel Screening Instrument for Psychosis (BSIP): development, structure, reliability and validity]. Fortschr Neurol Psychiatr, 76(4):207-216.

Smieskova R, Fusar-Poli P, Allen Pet al., 2009. The effects of antipsychotics on the brain: what have we learnt from structural imaging of schizophrenia?--a systematic review. Curr. Pharm. Des, 15(22):2535-2549.

Smieskova R, Fusar-Poli P, Allen P, et al., 2010. Neuroimaging predictors of transition to psychosis--a systematic review and meta-analysis. Neurosci Biobehav Rev, 34(8):12071222.

Tzourio-Mazoyer, N., Landeau, B., Papathanassiou, D., et al., 2002. Automated anatomical labeling of activations in SPM using a macroscopic anatomical parcellation of the MNI MRI single-subject brain. NeuroImage 15 (1), 273-289.

Yung AR, McGorry PD, McFarlane CA, et al., 1996. Monitoring and care of young people at incipient risk of psychosis. Schizophr Bull, 22(2):283-303.

Yung AR, Phillips LJ, McGorry PD, et al., 1998. Prediction of psychosis. A step towards indicated prevention of schizophrenia. Br J Psychiatry Suppl, 172(33):14-20.

Yung AR, Phillips LJ, Yuen HP, et al., 2003. Psychosis prediction: 12-month follow up of a high-risk ("prodromal”) group. Schizophr. Res., 60(1):21-32.

Yung AR, Yuen HP, Berger G, et al., 2007. Declining transition rate in ultra high risk (prodromal) services: dilution or reduction of risk? Schizophr Bull, 33(3):673-681.

Yung AR, Nelson B, Stanford C, et al., 2008. Validation of "prodromal" criteria to detect individuals at ultra high risk of psychosis: 2 year follow-up. Schizophr. Res., 105(1-3):1017.

Ventura J, Lukoff D, Nuechterlein KH, et al., 1993. Training and quality assurance with the brief psychiatric rating scale:__The Drift Busters__; Appendix 1 The Brief Psychiatric Rating Scale (expanded version). Int J Meth Psychiatric Res, 3:221-224.

Zarogianni, E., Moorhead, T.W.J., Lawrie, S.M., 2013. Towards the identification of imaging biomarkers in schizophrenia, using multivariate pattern classification at a singlesubject level. Neuroimage Clin. 3, 279-289.

Zarogianni E, Storkey AJ, Johnstone EC, et al., 2016. Improved individualized prediction of schizophrenia in subjects at familial high risk, based on neuroanatomical data, schizotypal and neurocognitive features. Schizophr Res. 
Figure 1. Representation of the nested LOO-CV SMV-RFE method. We employed a nested LOO-CV where we repeatedly excluded one subject to comprise the testing set and the remaining subjects were again repeatedly repartitioned in an internal validation loop where one subject was left out for validation and the rest formed the internal training group. In this loop, RFE was repeatedly performed and the mean accuracy on the validation group at each elimination level was recorded until all features were removed. The feature set that produced the maximum accuracy on the validation set was selected and applied to the testing set of the outer testing loop. Finally, mean accuracy was calculated across all outer CV loops.

Figure 2. Discrimination maps for the classification of ARMS-T vs ARMS-NT. The colours represent the weight of each feature in the classification function (the red scale represents positive weights and the blue scale represents negative weights). The SVM weight vector is a linear combination or weighted average of the support vectors and defines the decision boundary. The weight vector is therefore a spatial representation of the decision boundary. Every feature contributes with a certain weight to the decision boundary or classification function. Given a positive and a negative class (+1=ARMS-T; $-1=$ ARMS-NT group), a positive weight means the weighted average in that region was higher for the ARMS-T group, and a negative weight means the weighted average was higher for ARMS-NT group. Note: features correspond to GM volume measures in the AAL-defined brain regions, and not voxels. 
Table 1. ARMS inclusion and transition to psychosis criteria.

\section{ARMS Inclusion criteria \\ Inclusion into the study was based on the BSIP checklist and required one or more of the following:}

1. Attenuated psychotic-like symptoms: at least several times a week and for more than 1 week duration (a score of 2 or 3 on the Brief Psychiatric Rating Scale (BPRS)

2. Brief limited intermittent psychotic symptoms (BLIPS): scores of 4 or above on the hallucination item or 5 or above on the unusual thought content, suspiciousness, or conceptual disorganization items of the BPRS, with each symptom lasting less than 1 week before resolving spontaneously

3. Genetic risk: a first or second-degree relative with a psychotic disorder plus at least 2 further risk factors for or indicators of beginning psychosis according to the BSIP screening instrument.

\section{Criteria for transition to psychosis}

1. BPRS scores of 4 or above on the hallucination item or scores of 5 or above on the unusual thought content, suspiciousness, or conceptual disorganization items

2. Symptoms had to occur daily and persist for more than 1 week.

BSIP, Basel Screening Instrument for Psychosis; BPRS, Brief Psychiatric Rating Scale 
Table 2. Socio-demographic and clinical information of the ARMS-T and ARMS-NT study groups

\section{Study Groups}

\begin{tabular}{|c|c|c|c|}
\hline & & \multirow[b]{2}{*}{$P$} \\
\hline & ARMS-T & ARMS-NT & \\
\hline \multicolumn{4}{|l|}{ Socio-demographic variables } \\
\hline $\mathrm{N}$ & 16 & 19 & \\
\hline Mean age at baseline, $y(s d)$ & $26.8(6.5)$ & $23.9(6.2)$ & $\mathrm{ns}^{\mathrm{a}}$ \\
\hline Sex (male), n (\%) & $11(69)$ & $10(52)$ & $\mathrm{ns}^{\mathrm{b}}$ \\
\hline Educational level & & & $\mathrm{ns}^{\mathrm{b}}$ \\
\hline$<9 \mathrm{y}, \mathrm{n}(\%)$ & 3 & 8 & \\
\hline $9-11$ y, n (\%) & 7 & 7 & \\
\hline $12-13$ y, n (\%) & 5 & 2 & \\
\hline$<13 \mathrm{y}, \mathrm{n}(\%)$ & 1 & 2 & \\
\hline Mean verbal IQ (MWT-B) (sd) & $109.6(12.6)$ & $107.3(15.4)$ & $\mathrm{ns}^{\mathrm{a}}$ \\
\hline Cannabis use at baseline & & & $\mathrm{ns}^{\mathrm{b}}$ \\
\hline none & 10 & 11 & \\
\hline rarely & 1 & 1 & \\
\hline Several times/month & 0 & 2 & \\
\hline Several times/week & 4 & 0 & \\
\hline daily & 1 & 5 & \\
\hline Antipsychotics before entry, $\mathrm{n}(\%)$ & $6(37.5)$ & $1(5)$ & $<0.05^{\mathrm{b}}$ \\
\hline Antidepressants at baseline, $\mathrm{n}(\%)$ & $7(44)$ & $5(26)$ & $\mathrm{ns}^{\mathrm{b}}$ \\
\hline Family History & & & $\mathrm{ns}^{\mathrm{b}}$ \\
\hline No relative & 15 & 16 & \\
\hline One 1rst degree & 1 & 2 & \\
\hline One $2^{\text {nd }}$ degree & 0 & 1 & \\
\hline \multicolumn{4}{|l|}{ Clinical variables } \\
\hline Mean BPRS total score at baseline (sd) & $42.3(10.6)$ & $35.7(7.1)$ & $\mathrm{ns}^{\mathrm{c}}$ \\
\hline $\begin{array}{l}\text { Mean SANS global score at } \\
\text { baseline (sd) }\end{array}$ & $9.75(5.8)$ & $7.7(4.2)$ & $\mathrm{ns}^{\mathrm{c}}$ \\
\hline $\begin{array}{l}\text { Mean interval between MRI and } \\
\text { disease onset, } \mathrm{d}(\mathrm{sd})\end{array}$ & $306.3(318.3)$ & na & \\
\hline
\end{tabular}

ARMS-T: at-risk mental state individuals that later developed psychosis; ARMS-NT: atrisk mental state subjects that did not make a transition. BPRS: the Brief Psychiatric Rating Scale; SANS: the Scale for the Assessment of Negative Symptoms. Verbal IQ Mehrfach-Wortwahl-Test-B 
${ }^{a}$ Student's $T$-test. ${ }^{b}$ Fisher's exact test. ${ }^{c}$ Mann-Whitney U-test

Table 3. Classification performance

\begin{tabular}{|l|l|l|l|l|c|c|c|c|c|c|c|}
\hline & TP & TN & FP & FN & $\begin{array}{c}\text { Sens } \\
(\%)\end{array}$ & $\begin{array}{c}\text { Spec } \\
(\%)\end{array}$ & $\begin{array}{c}\text { BAC } \\
(\%)\end{array}$ & $\begin{array}{c}\text { FPR } \\
(\%)\end{array}$ & $\begin{array}{c}\text { PPV } \\
(\%)\end{array}$ & $\begin{array}{c}\text { NPV } \\
(\%)\end{array}$ & LR+/LR- \\
\hline $\begin{array}{l}\text { ARMS-T vs } \\
\text { ARMS-NT }\end{array}$ & 10 & 16 & 3 & 6 & 62.5 & 84.2 & 74.2 & 15.7 & 77 & 73 & $3.9 / 0.45$ \\
\hline
\end{tabular}

The diagnostic performance was evaluated by means of sensitivity (Sens), specificity (Spec), balanced accuracy (BAC), false positive rate (FPR) and positive/negative predictive value (PPV/NPV). LR+ was also calculated as sensitivity/1-specificity and LR- $=1$ sensitivity/specificity. 
Table 4. Misclassification analysis

\begin{tabular}{|c|c|c|c|c|c|c|}
\hline & $\begin{array}{l}\text { ARMS-T } \rightarrow \\
\text { ARMS-NT }\end{array}$ & $\begin{array}{l}\text { ARMS-T } \rightarrow \\
\text { ARMS-T }\end{array}$ & $P$ & $\begin{array}{l}\text { ARMS-NT } \rightarrow \\
\text { ARMS-T }\end{array}$ & $\begin{array}{l}\text { ARMS-NT } \rightarrow \\
\text { ARMS-NT }\end{array}$ & $P$ \\
\hline \multicolumn{7}{|l|}{$\begin{array}{l}\text { Socio-demographic } \\
\text { variables }\end{array}$} \\
\hline $\mathrm{N}$ & 6 & 10 & & 3 & 16 & \\
\hline $\begin{array}{l}\text { Mean age at baseline, y } \\
\text { (sd) }\end{array}$ & $29.2(9)$ & $25.4(4.5)$ & $\mathrm{ns}^{\mathrm{a}}$ & $24.8(7.2)$ & $23.8(6.2)$ & $\mathrm{ns}^{\mathrm{a}}$ \\
\hline Sex (male), n (\%) & $2(33)$ & $9(90)$ & $<0.05^{\mathrm{b}}$ & $1(33)$ & $9(56)$ & $n s^{b}$ \\
\hline Educational level & & & $n s^{\mathrm{c}}$ & & & $\mathrm{ns}^{\mathrm{c}}$ \\
\hline$<9 \mathrm{y}, \mathrm{n}(\%)$ & 1 & 2 & & 2 & 6 & \\
\hline $9-11$ y, n (\%) & 2 & 5 & & 1 & 6 & \\
\hline $12-13 \mathrm{y}, \mathrm{n}(\%)$ & 2 & 3 & & 0 & 2 & \\
\hline$<13 \mathrm{y}, \mathrm{n}(\%)$ & 1 & 0 & & 0 & 2 & \\
\hline Cannabis use at baseline & & & $\mathrm{ns}^{\mathrm{c}}$ & & & $\mathrm{ns}^{\mathrm{c}}$ \\
\hline none & 4 & 6 & & 2 & 9 & \\
\hline rarely & 1 & 0 & & 0 & 1 & \\
\hline Several times/month & 0 & 0 & & 1 & 1 & \\
\hline Several times/week & 1 & 3 & & 0 & 0 & \\
\hline daily & 0 & 1 & & 0 & 5 & \\
\hline $\begin{array}{l}\text { Antipsychotics before } \\
\text { entry }\end{array}$ & 5 & 1 & $<0.05^{\mathrm{b}}$ & 0 & 1 & $\mathrm{~ns}^{\mathrm{b}}$ \\
\hline $\begin{array}{c}\text { Anti-depressants at } \\
\text { baseline }\end{array}$ & 3 & 4 & $\mathrm{~ns}^{\mathrm{b}}$ & 2 & 3 & $\mathrm{~ns}^{\mathrm{b}}$ \\
\hline \multicolumn{7}{|l|}{ Clinical variables } \\
\hline $\begin{array}{l}\text { Mean BPRS total score } \\
\text { at baseline (sd) }\end{array}$ & $45.7(11.5)$ & $40.2(10.2)$ & $n s^{c}$ & $38.3(12.9)$ & $37.5(6.2)$ & $\mathrm{ns}^{\mathrm{c}}$ \\
\hline $\begin{array}{l}\text { Mean SANS global } \\
\text { score at baseline (sd) }\end{array}$ & $9.7(7.7)$ & $9.8(4.8)$ & $n s^{c}$ & $10.3(5)$ & $6.3(4.7)$ & $\mathrm{ns}^{\mathrm{c}}$ \\
\hline $\begin{array}{c}\text { Mean interval between } \\
\text { MRI and disease onset, } \\
\mathrm{d}(\mathrm{sd})\end{array}$ & $427.5(483.6)$ & $245.7(215.3)$ & $\mathrm{ns}^{\mathrm{a}}$ & & & \\
\hline
\end{tabular}

${ }^{a}$ Student's $T$-test. ${ }^{b}$ Fisher's exact test. ${ }^{\mathrm{c}}$ Mann-Whitney U-test 
Table 5. List of the most discriminative regions for the classification of ARMS-T vs ARMS-NT.

\begin{tabular}{cll}
\hline Lobe & Region/Hemisphere & w \\
\hline
\end{tabular}

\section{Negative weights}

Cerebellum

Temporal

$\begin{array}{lr}\text { Cerebellum_Crus2_R } & -0.0128 \\ \text { Cerebellum_3_R } & -0.0194 \\ \text { Cerebellum_4_5_R } & -0.0207 \\ \text { Cerebellum_6_L } & -0.0127 \\ \text { Cerebellum_7b_R } & -0.0146 \\ \text { Cerebellum_10_L } & -0.0247 \\ \text { Vermis_8 } & -0.0147\end{array}$

Temporal_Sup_R $\quad-0.0067$

Temporal_Pole_Sup_L $\quad-0.0084$

Temporal_Mid_L $\quad-0.0075$

Frontal

Parietal

Frontal_Sup_L $\quad-0.0197$

Frontal_Sup_Orb_L $\quad-0.0166$

Frontal_Mid_R $\quad-0.0089$

Frontal_Inf_Tri_R $\quad-0.0106$

Frontal_Sup_Medial_R $\quad-0.0084$

Frontal_Med_Orb_R $\quad-0.0098$

Precentral_R $\quad-0.0176$

$\begin{array}{llr} & \text { Postcentral_R } & -0.0102 \\ \text { Limbic } & \text { Paracentral_Lobule_R } & -0.012 \\ & \text { Cingulum_Ant_R } & -0.017 \\ \text { Basal ganglia } & \text { Cingulum_Post_L } & -0.0081 \\ \text { Perisylvian } & \text { Putamen_R } & -0.0172 \\ & \text { Insula_L } & -0.0134 \\ \text { Positive weights } & \text { Insula_R } & -0.0206 \\ \text { Temporal } & & \\ & \text { Temporal_Mid_R } & 0.0069 \\ \text { Frontal } & \text { Heschl_R } & 0.0069 \\ \text { Parietal } & \text { Frontal_Sup_Orb_R } & 0.0101 \\ \text { Basal ganglia } & \text { Parietal_Inf_R } & 0.0137 \\ & & \end{array}$




\section{Pallidum_L 0.0072}

Ant, anterior; Crus, crust; Inf, inferior; L, left hemisphere; Mid, middle; Med, medial; Orb, orbital; Post, posterior; R, right hemisphere; Sup, superior; w, weight vector of corresponding features in the classification process. Note: The SVM weight vector is a linear combination or weighted average of the support vectors and defines the decision boundary. The weight vector is therefore a spatial representation of the decision boundary. Every feature contributes with a certain weight to the decision boundary or classification function. Given a positive and a negative class (+1=ARMS-T; -1=ARMS-NT group), a positive weight means the weighted average in that voxel was higher for the ARMS-T group, and a negative weight means the weighted average was higher for ARMS-NT group. 

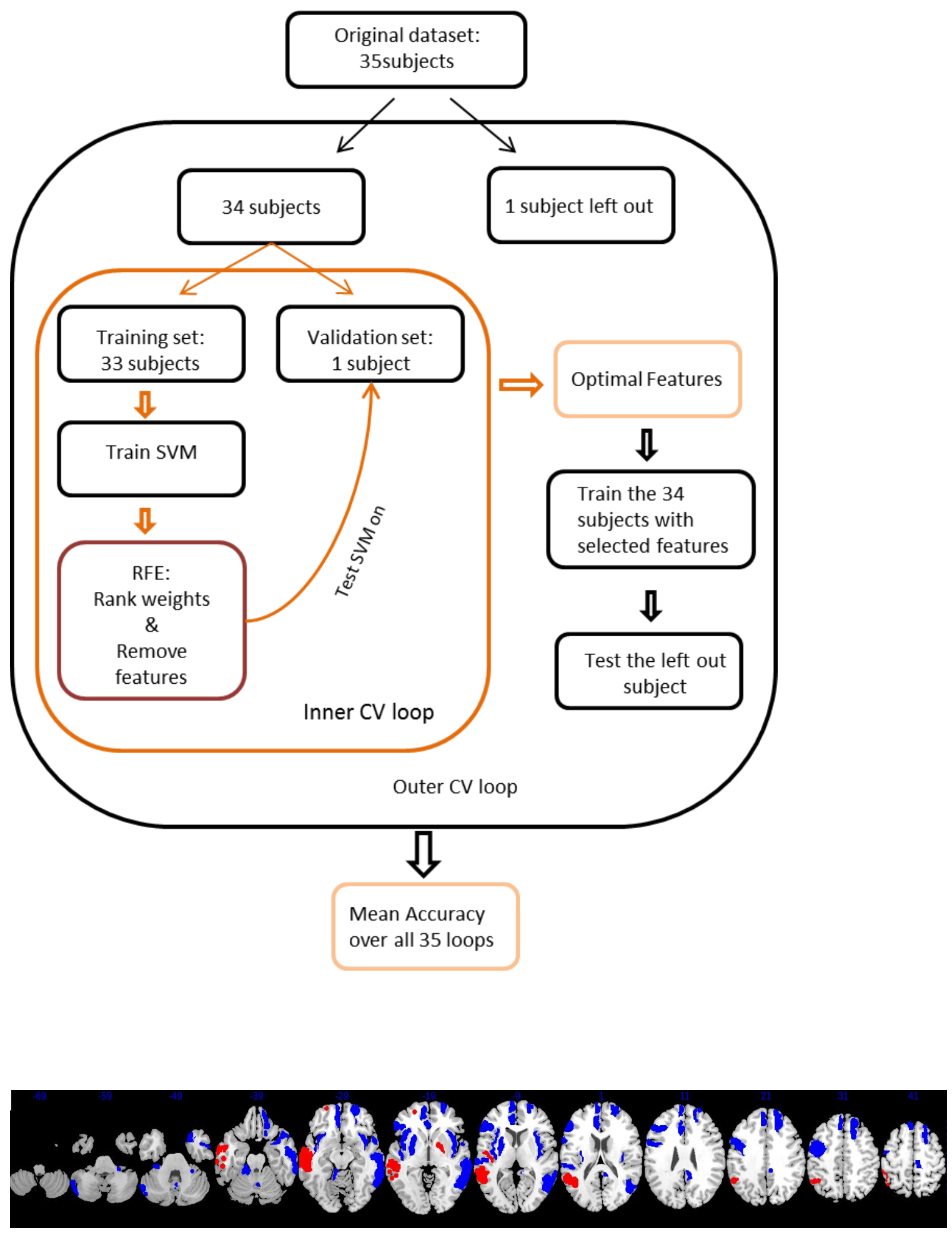